\title{
Bounds for the first eigenvalue of the elastically supported membrane on convex domains
}

\section{Journal Article}

\section{Author(s):}

Sperb, René Peter

Publication date:

2003-09

Permanent link:

https://doi.org/10.3929/ethz-b-000053592

\section{Rights / license:}

In Copyright - Non-Commercial Use Permitted

Originally published in:

Zeitschrift für angewandte Mathematik und Physik 54(5), https://doi.org/10.1007/s00033-003-3212-3 


\section{Bounds for the first eigenvalue of the elastically supported membrane on convex domains}

R. Sperb

To Larry Payne on his eightieth birthday

Abstract. Barta's principle and gradient bounds for the torsion function are the main tools for deriving lower bounds for the first eigenvalue. The optimal domains are an infinite strip, a disk or an annulus in different situations.

Mathematics Subject Classification (2000). 35P15.

Keywords. Eigenvalues of the Laplacian, Robin boundary conditions.

\section{Introduction}

The eigenvalue problem

$$
\begin{cases}\Delta u+\lambda u=0 & \text { in } \Omega \\ \frac{\partial u}{\partial n}+h u=0 & \text { on } \partial \Omega\end{cases}
$$

is usually called the "elastically supported membrane". The constant $h>0$ plays the role of a spring constant, with $h=0$ corresponding to a free boundary and $h=\infty$ describing a clamped boundary. In applications a probably more important aspect is the connection with diffusion problems with radiation at the boundary. In all cases however a lower bound for the first eigenvalue $\lambda_{1}(h)$ is of main interest, and many results have appeared in the literature.

In this paper lower bounds are derived for domains with boundary of positive mean curvature. The method used is an adaption of earlier works of Payne \& Philippin [9] and the author [17].

The main idea is to find a good function $v$ in Barta's principle stating that if $v \geq 0$ in $\Omega$ and

$$
\Delta v+\mu v \leq 0 \text { in } \Omega, \frac{\partial v}{\partial n}+h v \geq 0 \text { on } \partial \Omega
$$


then $\lambda_{1} \geq \mu$.

In the choice of the function $v$ the solution of the torsion problem

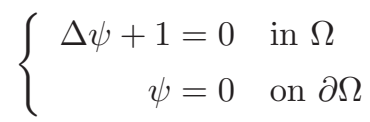

will play a central role as an auxiliary function. There are other choices of auxiliary problems. The torsion problem has the advantage that many bounds are known for the quantities relevant for the application to problem (1.1). In addition in two dimensions sharper results are known. This is also true if problem (1.1) and (1.3) are considered on a surface, which is equivalent to the "inhomogeneous membrane"

$$
\begin{cases}\Delta u+\lambda \rho(x) u=0 & \text { in } \Omega \\ \frac{\partial u}{\partial n}+h m(x) u=0 & \text { on } \partial \Omega\end{cases}
$$

with positive functions $\rho(x), m(x)$. In this case the torsion problem (1.3) has to be replaced by the Poisson problem

$$
\left\{\begin{aligned}
\Delta p+\rho=0 & \text { in } \Omega \\
p=0 & \text { on } \partial \Omega .
\end{aligned}\right.
$$

The lower bounds for $\lambda_{1}(h)$ derived in Theorems 3.1, 3.2, 3.3, 5.1 are all consequences of Lemma 2.1 in which a bound for $|\nabla \psi|^{2}$ or $\frac{1}{\rho}|\nabla p|^{2}$ is the basis. Our main concern here are plane domains, but the bound given in Theorem 3.3 is valid in higher dimensions as well. Theorem 3.2 is due to Payne \& Philippin [9], who proved it for the fixed membrane $(h \rightarrow \infty)$. Theorem 4.1 was proven by Pólya-Szegö [13] for the fixed membrane. The version for the elastically supported membrane is given in C. Bandle [2]. It is repeated here in order to compare with the different bounds. In Section 6 some numerical values are listed in order to give some idea of how close these bounds may get.

\section{Construction of a function $v(x)$}

The basic construction of a function $v(x)$ for Barta's principle is first developed. It will be used later on in four different cases.

Let $x$ be a generic point of $\Omega$ and set

$$
t(x)=\varepsilon\left(\psi_{m}-\psi(x)\right),
$$

with $\varepsilon$ a parameter to be chosen later and $\psi$ the solution of (1.3). We then set

$$
s(x)=\varepsilon^{-\frac{1}{2}} \cdot f(t(x)),
$$


where the function $f$ can be selected also. From (2.2) it follows that

$$
\nabla s=-\varepsilon^{\frac{1}{2}} \frac{d f}{d t} \cdot \nabla \psi .
$$

It is convenient to introduce the (dimensionless) variable

$$
\sigma=\varepsilon^{\frac{1}{2}} \cdot s .
$$

It is now assumed that we can write for some $A(\sigma)$

$$
\nabla s=-\varepsilon^{\frac{1}{2}} \cdot A(\sigma) \cdot \nabla \psi,
$$

so that

$$
\Delta s=\varepsilon^{\frac{1}{2}} \cdot A(\sigma)-\varepsilon^{\frac{3}{2}} A^{\prime}(\sigma) \cdot|\nabla \psi|^{2} .
$$

Hence the function $v(x)=X(s(x))$ satisfies

$$
\begin{aligned}
\Delta v+\mu v & =\frac{d X}{d s} \Delta s+\frac{d^{2} X}{d s^{2}} \cdot|\nabla s|^{2}+\mu X \\
& =\varepsilon^{\frac{1}{2}} A \cdot \frac{d X}{d s}\left[1+\varepsilon \frac{A^{\prime}}{A}|\nabla \psi|^{2}\right]+\frac{d^{2} X}{d s^{2}} \varepsilon A^{2} \cdot|\nabla \psi|^{2}+\mu X .
\end{aligned}
$$

We further assume that $X(s)$ satisfies

$$
\frac{d^{2} X}{d s^{2}}+a(s) \frac{d X}{d s}+\mu b(s) X=0,
$$

where $a(s), b(s)$ will be chosen below. Then the combination of (2.7) and (2.8) yields

$$
\Delta v+\mu v=\varepsilon^{\frac{1}{2}} A \frac{d X}{d s}\left[1+\left(\varepsilon \frac{A^{\prime}}{A}-\varepsilon^{\frac{1}{2}} a A\right)|\nabla \psi|^{2}\right]+\mu X\left[1-\varepsilon A^{2} b \cdot|\nabla \psi|^{2}\right]
$$

At this point it is assumed that a gradient bound is known, written in the form

$$
\varepsilon|\nabla \psi|^{2} B(\sigma) \leq 1
$$

Then we choose $a(\sigma), b(\sigma)$ such that

$$
\begin{aligned}
-\frac{A^{\prime}}{A}+a A & =B, \\
b A^{2} & =B,
\end{aligned}
$$

which allows to write

$$
\Delta v+\mu v=\left\{\sqrt{\varepsilon} X^{\prime}(s) A(\sigma)+\mu X(s)\right\}\left[1-\varepsilon B(\sigma)|\nabla \psi|^{2}\right] .
$$

By (2.10) the []-term is nonnegative. We now have to ensure that the \{\} -term is nonpositive. In terms of the variable $\sigma$ the differential equation (2.8) is with the selection (2.11), (2.12)

$$
X^{\prime \prime}+\left(\frac{A^{\prime}}{A}+\frac{B}{A}\right) X^{\prime}+\frac{\mu}{\varepsilon} \frac{B}{A^{2}} X=0 .
$$


This can be written in selfadjoint form as

$$
\left(p(\sigma) X^{\prime}\right)^{\prime}+\frac{\mu}{\varepsilon} q(\sigma) X=0,
$$

with $p(\sigma)=e(\sigma) A(\sigma), q(\sigma)=\frac{B}{A} e(\sigma)$, where

$$
e(\sigma)=\exp \left(\int \frac{B}{A}\right)
$$

and the integral is chosen such that $e(0)=0$. In addition to the differential equation (2.15) we select the boundary conditions

$$
X^{\prime}(0)=0, X^{\prime}\left(\sigma_{0}\right)+h \cdot \varepsilon^{-\frac{1}{2}} \frac{B^{\frac{1}{2}}}{A} X\left(\sigma_{0}\right)=0,
$$

with $\sigma_{0}=f\left(\varepsilon \psi_{m}\right)$.

We can now prove that the \{\} -term in (2.13) is nonpositive. To this end consider the function

$$
g(\sigma)=\left(\varepsilon X^{\prime}(\sigma) A(\sigma)+\mu X(\sigma)\right) e(\sigma) .
$$

Then $g(0)=0$ and

$$
\begin{aligned}
g^{\prime}(\sigma) & =\varepsilon\left(p \cdot X^{\prime}\right)^{\prime}+\mu(X e)^{\prime} \\
& =\varepsilon\left(p X^{\prime}\right)^{\prime}+\mu q X+\mu e(\sigma) \cdot X^{\prime}=\mu e(\sigma) X^{\prime} .
\end{aligned}
$$

But $p(\sigma) \geq 0, q(\sigma) \geq 0$, so that (2.15) implies immediately that $X^{\prime} \leq 0$ if $\mu$ is the first eigenvalue of (2.15) with corresponding positive eigenfunction $X(\sigma)$.

Hence we have $g(\sigma) \leq 0$, that is

$$
\Delta v+\mu v \leq 0 \text { in } \Omega .
$$

To check the boundary inequality of (1.2) we compute (using (2.5)

$$
\frac{\partial v}{\partial n}+h v=\frac{d X}{d s} \cdot \frac{\partial s}{\partial n}+h \cdot X=\frac{d X}{d s} \varepsilon^{\frac{1}{2}} \cdot A(\sigma)|\nabla \psi|+h \cdot X,
$$

and by (2.17) we see that

$$
\frac{\partial v}{\partial n}+h v=h \cdot X\left[1-\varepsilon^{\frac{1}{2}} \cdot B^{\frac{1}{2}} \cdot|\nabla \psi|\right] \geq 0,
$$

where the inequality is again a consequence of (2.10).

We can now summarize as follows

Lemma 2.1. Let $\psi$ be the solution of (1.3) and $\psi_{m}=\max _{\Omega} \psi(x)$. Let $\varepsilon$ be a positive parameter, $f(t)$ a nonnegative function and suppose $\frac{d f}{d t}=A(\sigma)$ with $t=\varepsilon\left(\psi_{m}-\psi\right), \sigma=f(t)$.

Suppose that $\varepsilon|\nabla \psi|^{2} B(\sigma) \leq 1$. 
Let $X(\sigma)$ be the first eigenfunction of

$$
\begin{aligned}
& \left(p(\sigma) X^{\prime}\right)^{\prime}+\frac{\mu}{\varepsilon} q(\sigma) X=0 \text { in }\left(0, \sigma_{0}\right) \\
& X^{\prime}(0)=0, X^{\prime}\left(\sigma_{0}\right)+h \cdot \varepsilon^{-\frac{1}{2}} \frac{B^{\frac{1}{2}}\left(\sigma_{0}\right)}{A\left(\sigma_{0}\right)} X\left(\sigma_{0}\right)=0,
\end{aligned}
$$

with $\sigma_{0}=f\left(\varepsilon \psi_{m}\right), p(\sigma)=e(\sigma) A(\sigma), q(\sigma)=\frac{B(\sigma)}{A(\sigma)} e(\sigma), e(\sigma)=\exp \left(\int \frac{B}{A}\right)$, $e(0)=0$.

Then the first eigenvalue of (1.1) satisfies

$$
\lambda_{1} \geq \frac{\mu_{1}}{\varepsilon} .
$$

For later on it is also convenient to state

Lemma 2.2. The first eigenvalue $\mu_{1}$ of

$$
\begin{aligned}
& \left(p(\sigma) X^{\prime}\right)^{\prime}+\mu q(\sigma) X=0 \text { in }\left(0, \sigma_{0}\right) \\
& X^{\prime}(0)=0, X^{\prime}\left(\sigma_{0}\right)+h \cdot X\left(\sigma_{0}\right)=0
\end{aligned}
$$

is decreasing with increasing length $\sigma_{0}$ of the interval $(p>0, q>0, h>0)$.

Proof.This is an immediate consequence of Rayleigh's principle for $\mu_{1}$ :

Assume $\sigma_{1}>\sigma_{0}$. Then

$$
\mu_{1}\left(\sigma_{1}\right)=\inf _{v} \frac{\int_{0}^{\sigma_{1}} p \cdot v^{\prime 2} d \sigma+h \cdot v^{2}\left(\sigma_{1}\right)}{\int_{0}^{\sigma_{1}} q v^{2} d \sigma} .
$$

Choose

$$
v(\sigma)= \begin{cases}u(\sigma), & 0 \leq \sigma \leq \sigma_{0} \\ u\left(\sigma_{0}\right), & \sigma_{0}<\sigma \leq \sigma_{1}\end{cases}
$$

where $u(\sigma)$ is the first eigenfunction for $\left(0, \sigma_{0}\right)$. Then

$$
\mu_{1}\left(\sigma_{1}\right) \leq \frac{\int_{0}^{\sigma_{0}} p u^{\prime 2} d \sigma+h u^{2}\left(\sigma_{0}\right)}{\int_{0}^{\sigma_{0}} q u^{2} d \sigma+u^{2}\left(\sigma_{0}\right) \int_{\sigma_{0}}^{\sigma_{1}} q d \sigma} \leq \frac{\int_{0}^{\sigma_{0}} p u^{\prime 2} d \sigma+h u^{2}\left(\sigma_{0}\right)}{\int_{0}^{\sigma_{0}} q u^{2} d \sigma}=\mu_{1}\left(\sigma_{0}\right) .
$$

Remark. In contrast to the eigenvalue $\lambda_{1} \equiv \lambda_{1}(\infty)$ of the fixed membrane the eigenvalue $\lambda(h)$ is not a monotone domain functional, i.e. if $\Omega \subset \widehat{\Omega}$ the associated eigenvalues do not necessarily satisfy $\lambda_{1}(h) \geq \widehat{\lambda}_{1}(h)$ (see also Payne-Weinberger [11]). A counter-example is given e.g. in Section 6 . 


\section{Applications of Lemma 1}

\subsection{Homogeneous membrane}

For the first result the starting point is a gradient bound of [14] stating that

$$
|\nabla \psi|^{2} \leq \frac{1}{\varepsilon}\left(1-\exp \left(-2 \varepsilon\left(\psi_{m}-\psi\right)\right)\right)
$$

with $\varepsilon=\frac{k_{0}}{\tau}, \tau=\max _{\partial \Omega}|\nabla \psi|$, curvature of $\partial \Omega=k \geq k_{0}$. For later applications it is important to note that one can always take $\varepsilon=\frac{k_{0}}{\bar{\tau}}$, with $\bar{\tau} \geq \tau$.

For $k_{0} \rightarrow 0$ (3.1) reduces to the bound of Payne [6]

$$
|\nabla \psi|^{2} \leq 2\left(\psi_{m}-\psi\right) \text {. }
$$

Now, for an interval $\left(-s_{0}, s_{0}\right)$ the solution of $(1.3)$ is

$$
\psi(x)=\frac{1}{2}\left(s_{0}^{2}-x^{2}\right)
$$

so that

$$
x=\left[2\left(\psi_{m}-\psi(x)\right)\right]^{\frac{1}{2}} .
$$

This leads to the choice

$$
s(x)=\left[2\left(\psi_{m}-\psi(x)\right)\right]^{\frac{1}{2}} .
$$

We now select $s(x)$ appropriate for the bound (3.1) and such that for $\varepsilon \rightarrow 0$ it reduces to $(3.3)$. Such a choice is

$$
s(x)=\varepsilon^{-\frac{1}{2}}\left[\exp \left(2 \varepsilon\left(\psi_{m}-\psi(x)\right)-1\right)\right]^{\frac{1}{2}},
$$

or in the notation of $(2.1)$

$$
f(t)=e^{2 t}-1
$$

Consequently one finds

$$
A(\sigma)=\frac{1+\sigma^{2}}{\sigma}
$$

and the bound (3.1) can be expressed in the form

$$
B(\sigma)=\frac{1+\sigma^{2}}{\sigma^{2}} .
$$

A short calculation shows that

$$
\begin{aligned}
& e(\sigma)=\sigma, \\
& p(\sigma)=1+\sigma^{2}, \\
& q(\sigma)=1,
\end{aligned}
$$


which means that the eigenvalue problem (2.21) is now

$$
\left\{\begin{array}{l}
\left(\left(1+\sigma^{2}\right) X^{\prime}\right)^{\prime}+\frac{\mu}{\varepsilon} X=0 \text { in }\left(0, \sigma_{0}\right) \\
X^{\prime}(0)=0, X^{\prime}\left(\sigma_{0}\right)+h \varepsilon^{-\frac{1}{2}}\left(1+\sigma_{0}^{2}\right)^{-\frac{1}{2}} X\left(\sigma_{0}\right)=0, \sigma_{0}=\left[e^{2 \varepsilon \psi_{m}}-1\right]^{\frac{1}{2}} .
\end{array}\right.
$$

The first result may be stated as

Theorem 3.1. Assume that the curvature $k$ of $\partial \Omega$ is bounded below by $k_{0}>0$ and $\max _{\Omega} \psi \leq \bar{\psi}_{m}, \max _{\partial \Omega}|\nabla \psi| \leq \bar{\tau}$. Set $\varepsilon=\frac{k_{0}}{\bar{\tau}}, \quad \nu=-\frac{1}{2}+i\left(\frac{\mu}{\varepsilon}-\frac{1}{4}\right)^{\frac{1}{2}}$, $\sigma_{0}=\left[e^{2 \varepsilon \bar{\psi}_{m}}-1\right]^{\frac{1}{2}}$. Then

$$
\lambda_{1}(h) \geq \mu_{1}(h)
$$

where $\mu_{1}$ is the first positive solution of

$$
-\frac{\mu \cdot \varepsilon^{-\frac{1}{2}} \operatorname{Im}\left[P_{\nu}^{-1}\left(i \cdot \sigma_{0}\right)\right]}{\operatorname{Re}\left[P_{\nu}\left(i \sigma_{0}\right)\right]}=h .
$$

$P_{\nu}, P_{\nu}^{-1}:$ Legendre functions.

Remark 3.1. For the quantities $\psi_{m}$ and $\tau$ one can take upper bounds $\bar{\psi}_{m}, \bar{\tau}$. This follows for $\psi_{m}$ from Lemma 2.2, for $\tau$ it is implied in the derivation of the bound (3.1).

Remark 3.2. Invariance of (3.11). A question that may arise is whether a different choice of a function $f(t)$ in (3.5) would lead to a different eigenvalue problem, and one could then try to optimize $f(t)$. This is not the case as the following calculations show.

With the abbreviations $t=\varepsilon\left(\psi_{m}-\psi\right)$ and $y=e^{t}$ assume that in the place of (3.4) we have

$$
s(x)=\varepsilon^{-\frac{1}{2}} \cdot f^{-1}(y)
$$

for some $f$. Then

$$
\nabla s=-\varepsilon^{\frac{1}{2}} \frac{d f^{-1}}{d y} \cdot y \cdot \nabla \psi
$$

but

$$
\frac{d f^{-1}}{d y}=\frac{1}{f^{\prime}(\sigma)}, \quad \sigma=\varepsilon^{\frac{1}{2}} \cdot s
$$

and therefore

$$
A(\sigma)=\frac{f(\sigma)}{f^{\prime}(\sigma)} .
$$

The gradient bound (3.1) can be written as

$$
B(\sigma)=\frac{1}{1-\frac{1}{y^{2}}}=\frac{y^{2}}{y^{2}-1}=\frac{f^{2}}{f^{2}-1} .
$$


A short calculation gives the associated differential equation (2.21) of Lemma 2.1 as

$$
\left(\frac{f}{f^{\prime}} \sqrt{f^{2}-1} \cdot X^{\prime}(\sigma)\right)^{\prime}+\frac{\mu}{\varepsilon} \frac{f^{\prime} f}{\sqrt{f^{2}-1}} X=0 .
$$

Choosing the new variable $z=\sqrt{f^{2}-1}$ one finds again

$$
\left(\left(1+z^{2}\right) X^{\prime}\right)^{\prime}+\frac{\mu}{\varepsilon} X=0 \text { in }\left(0, z_{0}\right),
$$

with $z_{0}=\sqrt{e^{2 \varepsilon \psi_{m}}-1}$ as in (3.11), and the boundary conditions are the same as well.

Remark 3.3. The change of variable $\sigma=\sinh (\sqrt{\varepsilon} s)$ transforms (3.11) into

$$
\begin{aligned}
& \left\{\begin{array}{l}
X^{\prime \prime}+\sqrt{\varepsilon} \tanh (\sqrt{\varepsilon} s) X^{\prime}+\mu X=0 \text { in }\left(0, s_{0}\right) \\
X^{\prime}\left(s_{0}\right)+h X\left(s_{0}\right)=0
\end{array}\right. \\
& s_{0}=\frac{1}{\sqrt{\varepsilon}} \operatorname{arch}\left(e^{\varepsilon \psi_{m}}\right) .
\end{aligned}
$$

This shows that for $\varepsilon \rightarrow 0$ one has $X(s)=\cos (\sqrt{\mu} s)$ and $s_{0}=\sqrt{2 \psi_{m}}$. In particular one is led to

$$
\lambda_{1} \geq \frac{\pi^{2}}{8 \psi_{m}}
$$

as proven by Payne [7] by different means.

The second result is a straightforward extension of an eigenvalue estimate of Payne \& Philippin [9] to the case of the elastically supported membrane. It is based on their gradient bound

$$
|\nabla \psi|^{2} \leq \varepsilon\left(\psi_{m}-\psi\right)
$$

where now

$$
\varepsilon=1+\left(1-\frac{2 M_{0}}{\psi_{m}}\right)^{\frac{1}{2}},
$$

with

$$
M_{0}=\min _{\partial \Omega}\left(k \cdot|\nabla \psi|^{3}\right)
$$

for a convex domain. In the case of an ellipse of semiaxes $a, b$ one has

$$
M_{0}=\frac{a^{4} b^{4}}{\left(a^{2}+b^{2}\right)^{3}}
$$

and

$$
\varepsilon=1+\frac{\left|a^{2}-b^{2}\right|}{a^{2}+b^{2}} .
$$


It follows from another gradient bound of Payne \& Philippin [8] that one may take

$$
M_{0}=\frac{k_{0}}{k_{m}^{3}}, \quad 0<k_{0} \leq k \leq k_{m}
$$

The important point is that with the choice (3.17) the equality sign holds in (3.14) if $\Omega$ is a disk or an infinite strip.

Choosing now

$$
s(x)=\sqrt{\varepsilon\left(\psi_{m}-\psi(x)\right)}
$$

one is led to

$$
p(s)=\frac{\varepsilon}{2} \cdot s^{\frac{2}{\varepsilon}-1}, q(s)=\frac{2}{\varepsilon} s^{\frac{2}{\varepsilon}-1}
$$

so that Lemma 2.1 leads to the eigenvalue problem

$$
\left\{\begin{array}{l}
\left(s^{\frac{2}{\varepsilon}-1} \cdot X^{\prime}\right)^{\prime}+\frac{4}{\varepsilon^{2}} \cdot s^{\frac{2}{\varepsilon}-1} \cdot \mu X=0 \text { in }\left(0, s_{0}\right) \\
X^{\prime}(0)=0, X^{\prime}\left(s_{0}\right)+\frac{2}{\varepsilon} h \cdot X\left(s_{0}\right)=0, s_{0}=\sqrt{\varepsilon \psi_{m}} .
\end{array}\right.
$$

In terms of the stretched variable $\sigma=\frac{2}{\varepsilon} s$ the solution of the differential equation is $X(\sigma)=\sigma^{\nu} \cdot J_{-\nu}(\sqrt{\mu} \sigma), J=$ Bessel function, $\nu=1-\frac{1}{\varepsilon}$ and the boundary condition at $\sigma_{0}=\sqrt{\frac{4 \psi_{m}}{\varepsilon}}$ becomes

$$
X^{\prime}\left(\sigma_{0}\right)+h \cdot X\left(\sigma_{0}\right)=0
$$

Note that the function $\sigma^{\nu} \cdot J_{-\nu}(\sigma)$ has the series expansion

$$
\sum_{k=0}^{\infty} \frac{(-1)^{k}}{k ! \Gamma(k+1-\nu)}\left(\frac{\sigma}{2}\right)^{2 k}
$$

Collecting now all facts and using identities for Bessel functions we may sum up in

Theorem 3.2. Assume that the curvature $k$ of $\partial \Omega$ satisfies $0<k_{0} \leq k \leq k_{m}$. Set

$$
\varepsilon=1+\sqrt{1-\frac{k_{0}}{4 \bar{\psi}_{m} \cdot k_{m}^{3}}}, \quad \nu=1-\frac{1}{\varepsilon}, \quad \sigma_{0}=\sqrt{\frac{4 \bar{\psi}_{m}}{\varepsilon}} .
$$

Then

$$
\lambda_{1}(h) \geq \mu_{1}(h)
$$

where $\mu_{1}(h)$ is the first positive solution of

$$
\sqrt{\mu} \frac{J_{1-\nu}\left(\sqrt{\mu} \sigma_{0}\right)}{J_{-\nu}\left(\sqrt{\mu} \sigma_{0}\right)}=h .
$$

The bound for $\lambda_{1}(h)$ is sharp if $\Omega$ is a disk or an infinite strip. 
Remark 3.4. The invariance property again holds for (3.19): one could choose in the place of (3.18) $s(x)=f\left[\varepsilon\left(\psi_{m}-\psi(x)\right)\right], f$ arbitrary, and Lemma 2.1 will again lead to (3.19).

Remark 3.5. An extension of the famous Faber-Krahn inequality for $\lambda_{1}(=$ $\left.\lambda_{1}(\infty)\right)$ to the case of the elastically supported membrane was proven by Bossel $[3]:$

One has $\lambda_{1}(h) \geq \mu_{1}(h)$ where $\mu_{1}(h)$ is the solution of

$$
\sqrt{\mu} \frac{J_{1}\left(\sqrt{\frac{\mu A}{\pi}}\right)}{J_{0}\left(\sqrt{\frac{\mu A}{\pi}}\right)}=h, \quad A=|\Omega| .
$$

A comparison of the bounds given by (3.20) or (3.21) is given in Section 6 .

\subsection{Domain $\Omega \subset \mathbb{R}^{N}, N \geq 2$}

Although the main interest is in plane domains, a result valid in higher dimensions as well is added here. The gradient bound in the present case is [14]

$$
|\nabla \psi|^{2} \leq 2\left(\psi_{m}-\psi\right) \frac{\alpha \psi+1}{\alpha \psi_{m}+1}
$$

with

$$
\alpha=\frac{(N-1) H_{0}}{\tau}, H=\text { mean curvature of } \partial \Omega \geq H_{0}>0 .
$$

Here it is again important for applications that one may take an upper bound $\bar{\tau}$ for $\tau$. We set

$$
\varepsilon=\frac{\alpha}{2\left(1+\alpha \psi_{m}\right)}
$$

and

$$
r(x)=\frac{\alpha\left(\psi_{m}-\psi(x)\right)}{\alpha \psi(x)+1} .
$$

We now define

$$
s(x)=\varepsilon^{-\frac{1}{2}} \cdot(r(x))^{\frac{1}{2}} .
$$

One finds then

$$
\nabla r=-2 \varepsilon(1+r)^{2} \nabla \psi
$$

and

$$
\nabla s=-\varepsilon^{\frac{1}{2}} \frac{\nabla r}{2 r^{\frac{1}{2}}}=-\varepsilon^{\frac{1}{2}} \frac{(1+r)^{2}}{r^{\frac{1}{2}}} \nabla \psi .
$$

Setting $\sigma=\varepsilon^{\frac{1}{2}} \cdot s$ we thus have in the notation of Lemma 2.1

$$
A(\sigma)=\frac{\left(1+\sigma^{2}\right)^{2}}{\sigma}, B(\sigma)=\frac{\left(1+\sigma^{2}\right)^{2}}{\sigma^{2}},
$$


which means that the eigenvalue problem (2.21) now takes the form

$$
\begin{aligned}
& \left\{\begin{array}{l}
\left(\left(1+\sigma^{2}\right)^{2} X^{\prime}\right)^{\prime}+\frac{\mu}{\varepsilon} X=0 \text { in }\left(0, \sigma_{0}\right) \\
X^{\prime}(0)=0,\left(1+\sigma_{0}^{2}\right) X^{\prime}\left(\sigma_{0}\right)+h \varepsilon^{-\frac{1}{2}} \cdot X\left(\sigma_{0}\right)=0
\end{array}\right. \\
& \text { with } \sigma_{0}=\left(\alpha \psi_{m}\right)^{\frac{1}{2}}
\end{aligned}
$$

The solution of (3.29) can be written in terms of Legendre functions $P_{\nu}^{m}$.

Theorem 3.3. Assume that the mean curvature $H$ of $\partial \Omega$ satisfies $H \geq H_{0}>0$.

Set $\alpha=\frac{(N-1) H_{0}}{\bar{\tau}}, \quad \varepsilon=\frac{\alpha}{2\left(1+\alpha \bar{\psi}_{m}\right)}$ where $\bar{\tau}, \bar{\psi}_{m}$ are upper bounds for the respective quantities.

Then one has

$$
\lambda_{1}(h) \geq \mu_{1}(h)
$$

where $\mu_{1}(h)$ is the first positive solution of

$$
\sqrt{\varepsilon}(1+\nu) \frac{\operatorname{Im} P_{0}^{\nu}\left(i \sigma_{0}\right)}{\operatorname{Re} P_{1}^{\nu}\left(i \sigma_{0}\right)}=h
$$

with $\nu=\sqrt{1+\frac{\mu}{\varepsilon}}, \sigma_{0}=\sqrt{\alpha \bar{\psi}_{m}}$.

Remark 3.6. Equation (3.30) is obtained by applying relations for the derivatives of Legendre functions (see e.g. [1], p. 334) and using the fact that the first eigenfunction of (3.29) is of the form

$$
X(\sigma)=\left(1+\sigma^{2}\right)^{-\frac{1}{2}} \cdot P_{1}^{\nu}(i \sigma), \nu=\sqrt{1+\frac{\mu}{\varepsilon}}
$$

Remark 3.7. Lemma 2.2 implies that an upper bound $\bar{\psi}_{m}$ for $\psi_{m}$ is sufficient.

Remark 3.8. The eigenvalue problem (3.29) is also invariant in the same sense as before. 


\section{Upper bounds for $\lambda_{1}(h)$}

\section{A. Extension of a bound of Pólya-Szegö}

Let $\Omega$ be a starshaped plane domain whose representation in polar coordinates $(r, \varphi)$ with respect to some origin is $r=R(\varphi)$. Set

$$
\begin{aligned}
B=\oint_{\partial \Omega} \frac{d s}{\vec{r} \cdot \vec{n}}, & \vec{r}=\text { radius vector, }|\vec{r}|=R(\varphi), \\
\vec{n} & =\text { exterior normal }
\end{aligned}
$$

and let $L$ be the length of $\partial \Omega, A$ the area of $\Omega$. The following immediate extension of a classical result of Pólya-Szegö [13] then holds (see also C. Bandle [2], p. 141):

Theorem 4.1. Set $\alpha=\frac{h \cdot L}{B}$. Then

$$
\lambda_{1}(h) \leq \mu_{1}(\alpha) \cdot \frac{B}{2 A}
$$

where $\mu_{1}(\alpha)$ is the first positive solution of

$$
\frac{\sqrt{\mu} \cdot J_{1}(\sqrt{\mu})}{J_{0}(\sqrt{\mu})}=\alpha .
$$

Equality holds in (4.2) if $\Omega$ is a disk.

Proof. For the convenience of the reader we repeat the steps of the proof which are essentially the same as in [13]. According to Rayleigh's principle one has

$$
\lambda_{1}(h) \leq \frac{\mathcal{D}(v)+h \oint_{\partial \Omega} v^{2} d s}{\int_{\Omega} v^{2} d x}, \mathcal{D}(v)=\text { Dirichlet's integral }
$$

Following Pólya-Szegö we use the trial function

$$
v=g\left(\frac{r}{R(\varphi)}\right)
$$

where $g$ is any $C^{1}$ function. Then one has

$$
\mathcal{D}(v)=\int_{0}^{\frac{\pi}{2}} \int_{0}^{R(\varphi)} g^{\prime 2}\left(\frac{1}{R^{2}(\varphi)}+\frac{\dot{R}^{2}}{R^{4}(\varphi)}\right) r d r d \varphi .
$$


Here we have used a prime for derivative of $g$ with respect to its variable and a dot for $\frac{d R}{d \varphi}$. Choosing the new variable $t=\frac{r}{R(\varphi)}$ the Dirichlet integral becomes

$$
\mathcal{D}(v)=\int_{0}^{2 \pi}\left(1+\frac{\dot{R}^{2}}{R^{2}}\right) d \varphi \int_{0}^{1} g^{\prime 2}(t) t d t .
$$

An elementary calculation shows that

$$
\int_{0}^{2 \pi}\left(1+\frac{\dot{R}^{2}}{R^{2}}\right) d \varphi=B
$$

Analogously one has

$$
\int_{\Omega} v^{2} d x=\int_{0}^{2 \pi} \int_{0}^{1} g^{2}(t) t d t R^{2}(\varphi) d \varphi=2 A \int_{0}^{1} g^{2}(t) t d t .
$$

Rayleigh's principle then tells us that

$$
\lambda_{1}(h) \leq \frac{B \int_{0}^{1} g^{\prime 2}(t) t d t+h \cdot L \cdot g^{2}(1)}{2 A \int_{0}^{1} g^{2}(t) t d t}=\frac{B}{2 A} \frac{\int_{0}^{1} g^{2}(t) t d t+\alpha g^{2}(1)}{\int_{0}^{1} g^{2}(t) t d t},
$$

but the second expression on the right is just the Rayleigh quotient for a radially symmetric solution on the unit circle. The optimal choice is known to be $g(t)=$ $J_{0}(\sqrt{\mu} t)$, where $\mu$ solves $(4.3)$.

\section{B. Application of an inequality of Payne \& Rayner}

It was shown in $[10]$ that the first eigenfunction $u_{1}$ in the fixed membrane problem satisfies

$$
\int_{\Omega} u_{1}^{2} d x \leq \frac{\lambda_{1}}{4 \pi}\left(\int_{\Omega} u_{1} d x\right)^{2},
$$

with equality if $\Omega$ is a disk. As an application of this inequality one finds the following upper bound (see [15]):

$$
\lambda_{1}(h) \leq \frac{\lambda_{1}}{2\left(\lambda_{1} A-4 \pi\right)}\left\{\lambda_{1} A+h \cdot L-\left[\left(\lambda_{1} A-h \cdot L\right]^{2}+16 \pi h \cdot L\right]^{\frac{1}{2}}\right\} .
$$

This inequality obtained by taking as a trial function in Rayleigh's principle $v=$ $u_{1}+c$, applying (4.4) and then optimizing the constant $c$. 
Remark 4.1. In some cases a slightly different version may also be useful: take any function $v$ satisfying $v=0$ for which the quantities

$$
d=\int_{\Omega}|\nabla v|^{2} d x, \quad n_{2}=\int_{\Omega} v^{2} d x, n_{1}=\int_{\Omega} v d x
$$

can be calculated. Then one finds analogously to (4.5)

$$
\lambda_{1}(h) \leq \frac{1}{2\left(n_{2} A-n_{1}^{2}\right)}\left\{n_{2} \cdot L \cdot h+d \cdot A-\left[\left(n_{2} \cdot h \cdot L-d \cdot A\right)^{2}+4 d \cdot h \cdot L \cdot n_{1}^{2}\right]^{\frac{1}{2}}\right\} .
$$

\section{Inhomogeneous membrane}

\subsection{Preliminary remarks}

The torsion problem on a surface $S$, i.e.,

$$
\widetilde{\Delta} \widetilde{\psi}+1=0 \text { in } \Omega \subset S, \widetilde{\psi}=0 \text { on } \partial \Omega,
$$

where we have used the notation $\widetilde{\Delta}$ for the Laplace-Beltrami operator of a surface, is equivalent to the Poisson problem (1.5). If the line element $d s$ of $S$ satisfies

$$
d s^{2}=\rho\left(x^{1}, x^{2}\right)\left(\left(d x^{1}\right)^{2}+\left(d x^{2}\right)^{2}\right),
$$

then

$$
\widetilde{\Delta} u=\frac{1}{\rho}\left(\frac{\partial^{2} u}{\left(\partial x^{1}\right)^{2}}+\frac{\partial^{2} u}{\left(\partial x^{2}\right)^{2}}\right)=\frac{1}{\rho} \Delta u
$$

and the normal derivative $\frac{\partial}{\partial \widetilde{n}}$ on $\partial \Omega$ in $S$ is

$$
\frac{\partial u}{\partial \widetilde{n}}=\frac{1}{\sqrt{\rho}} \frac{\partial u}{\partial n}
$$

Problem (1.4) can be interpreted as

$$
\begin{gathered}
\widetilde{\Delta}+\lambda \widetilde{u}=0 \text { in } \Omega \subset S, \\
\frac{\partial \widetilde{u}}{\partial \widetilde{n}}+h \cdot \widetilde{m} \widetilde{u}=0 \text { on } \partial \Omega \subset S,
\end{gathered}
$$

with $\widetilde{m}(x)=\frac{m(x)}{\sqrt{\rho}}$.

This correspondence between a problem on a surface and an inhomogeneous problem can be exploited in many ways (see e.g. [2], [16]). Later on we will need bounds for the gradient and the solution of the Poisson problem and we list some results of this type in the following.

For problem (5.1) it was shown in [16] that

$$
|\widetilde{\nabla} \widetilde{\psi}|^{2} \leq \frac{1}{K}\left(e^{2 K\left(\widetilde{\psi}_{m}-\widetilde{\psi}\right)}-1\right),
$$


provided that the Gaussian curvature $K_{G}$ of $S$ satisfies

$$
K_{G} \geq K>0,
$$

and

$$
k_{g}+K \cdot \widetilde{\tau} \geq 0 \text { on } \partial \Omega,
$$

where $k_{g}$ is the geodesic curvature of $\partial \Omega \subset S$ and

$$
\widetilde{\tau}=\max _{\partial \Omega}|\widetilde{\nabla} \widetilde{\psi}|=\max _{\partial \Omega} \frac{1}{\sqrt{\rho}}|\nabla \psi| .
$$

A sufficient criterion for the validity of (5.9) is

$$
K \cdot A+k_{g} \cdot L \geq 0,
$$

where $A=\int_{\Omega} \rho d x$ is the measure of $\Omega$ in $S$ and $L=\oint_{\partial \Omega} \sqrt{\rho} d s$ the measure of $\partial \Omega$ in $S$.

Equality holds in (5.7) if $\Omega$ is a geodesic strip $S_{g}$ on a sphere, i.e. the region represented in spherical coordinates by

$$
S_{g}:\left\{(\vartheta, \varphi), \frac{\pi}{2}-\beta<\vartheta<\frac{\pi}{2}+\beta, 0 \leq \varphi \leq 2 \pi, 0<\beta<\frac{\pi}{2}\right\} .
$$

For $S_{g}$ the solution of (5.1) is given by

$$
\widetilde{\psi}=R^{2} \cdot \log \left(\frac{\sin \vartheta}{\cos \beta}\right), R=\text { radius of the sphere } .
$$

Here of course $K_{G}=K=\frac{1}{R^{2}}$.

Finally we describe the correspondance between problem (5.1) and the geodesic strip $S_{g}$. First, the Gaussian curvature is

$$
K_{G}=-\frac{\Delta(\log \rho)}{2 \rho},
$$

and the geodesic curvature becomes

$$
k_{g}=\frac{1}{\sqrt{\rho}}\left(k+\frac{1}{2} \frac{\partial}{\partial n}(\log \rho)\right), k=\text { ordinary curvature of } \partial \Omega .
$$

Take now $\Omega$ as an annulus of radii $R_{1}, R_{2}$ and

$$
\rho=\rho(r)=\frac{1}{\left(1+\gamma r^{2}\right)^{2}} .
$$

Then $K_{G}=4 \gamma$ and the solution of (1.5) is

$$
p=\frac{1}{4 \gamma} \log \left(\frac{r\left(1+\gamma R_{1}^{2}\right)}{R_{1}\left(1+\gamma r^{2}\right)}\right),
$$

with $R_{1}>0$ and $R_{2}=\frac{1}{\gamma R_{1}}$. 
One then has

$$
\left(\frac{|\nabla p|^{2}}{\rho}+\frac{1}{K}\right) e^{2 K p}=\frac{\left(1+\gamma R_{1}^{2}\right)^{2}}{16 \gamma^{2} R_{1}^{2}}=\text { const. },
$$

and the maximum value is attained for $r=\frac{1}{\sqrt{\gamma}}$ and becomes

$$
p_{m}=\frac{1}{4 \gamma} \log \left(\frac{1+\gamma R_{1}^{2}}{2 \sqrt{\gamma} R_{1}}\right)=\frac{1}{2 K} \log \left(1+K \cdot \frac{A^{2}}{L^{2}}\right),
$$

with

$$
\begin{aligned}
& A=2 \pi \int_{R_{1}}^{\frac{1}{\gamma R_{1}}} \frac{r d r}{\left(1+\gamma r^{2}\right)^{2}}=\pi \frac{1-\gamma R_{1}^{2}}{\gamma\left(1+\gamma R_{1}^{2}\right)}, \\
& L=\frac{4 \pi R_{1}}{1+\gamma R_{1}^{2}} .
\end{aligned}
$$

In this case one also finds that

$$
K \cdot \widetilde{\tau}+k_{g}=K \cdot \frac{A}{L}+k_{g}=\frac{1}{r}(1-\gamma r+|1-\gamma r|) \geq 0 .
$$

\subsection{A lower bound for $\lambda_{1}(h)$ for (1.4)}

The calculations are completely analogous to the ones leading to Theorem 3.1: one just has to replace $\varepsilon$ there by $-K$. Hence setting now

$$
s(x)=K^{-\frac{1}{2}}\left[1-\exp \left(-2 K\left(p_{m}-p(x)\right)\right)\right]^{\frac{1}{2}},
$$

where $p$ is the solution of (1.5), and by equivalence, $p(x)=\widetilde{\psi}(x)$. In the notation of Section 2 one thus finds

$$
A(\sigma)=\frac{1-\sigma^{2}}{\sigma}
$$

and the bound (5.7) leads to

$$
B(\sigma)=\frac{1-\sigma^{2}}{\sigma^{2}}
$$

and similarly as in Section 3 the eigenvalue problem now is

$$
\begin{aligned}
& \left\{\begin{array}{l}
\left(\left(1-\sigma^{2}\right) X^{\prime}\right)^{\prime}+\frac{\mu}{K} X=0 \text { in }\left(0, \sigma_{0}\right) \\
X^{\prime}(0)=0, X^{\prime}\left(\sigma_{0}\right)+\widetilde{h} K^{-\frac{1}{2}}\left(1-\sigma_{0}^{2}\right)^{-\frac{1}{2}} X\left(\sigma_{0}\right)=0
\end{array}\right. \\
& \text { with } \sigma_{0}=\left[1-e^{-2 K p_{m}}\right]^{\frac{1}{2}} \text {. }
\end{aligned}
$$

One can check that $v(x)=X(s(x))$ satisfies

$$
\frac{\partial v}{\partial n}+h m(x) v \geq 0 \text { on } \partial \Omega
$$


if we choose $\widetilde{h}=h \cdot m_{0}, m_{0}=\min _{\partial \Omega} m(x)$.

The solution of (5.26) is given by

$$
X(\sigma)=P_{\nu}(\sigma) \cdot Q_{\nu-1}(0)+Q_{\nu}(\sigma) \cdot P_{\nu-1}(0), \quad \nu=-\frac{1}{2}+\sqrt{\frac{1}{4}+\frac{\mu}{K}},
$$

where $P, Q$ are Legendre functions, and summarizing one is led to

Theorem 5.1. Suppose the following inequalities hold:

(a) $-\frac{\Delta(\log \rho)}{2 \rho} \geq K>0$ in $\Omega$

(b) $\frac{1}{\sqrt{\rho}}\left(k+\frac{1}{2} \frac{\partial}{\partial n}(\log \rho)\right) \geq k_{g}$ on $\partial \Omega, k=$ curvature of $\partial \Omega$.

(c) $K \cdot A+k_{g} \cdot L \geq 0, A=\int_{\Omega} \rho d x, L=\oint_{\partial \Omega} \sqrt{\rho} d s$.

Set $\sigma_{0}=\left(1-\exp \left(-2 K \bar{p}_{m}\right)^{\frac{1}{2}}, \bar{p}_{m} \geq \max _{\Omega} p(x), p(x)=\right.$ solution of $(1.5)$ and

$$
\nu=-\frac{1}{2}+\sqrt{\frac{\mu}{K}+\frac{1}{4}}, \quad \beta=\frac{\sqrt{1-\sigma_{0}^{2}} \cdot h}{m_{0} \nu \sqrt{K}}, m_{0}=\min _{\partial \Omega} m(x) .
$$

Let $\mu_{1}(h)$ be the first positive solution of

$$
\frac{P_{\nu-1}\left(\sigma_{0}\right)+\left(\beta-\sigma_{0}\right) P_{\nu}\left(\sigma_{0}\right)}{Q_{\nu-1}\left(\sigma_{0}\right)+\left(\beta-\sigma_{0}\right) Q_{\nu}\left(\sigma_{0}\right)}=-\frac{2}{\pi} \cot \left(\frac{\pi}{2}(\nu-1)\right) .
$$

Then the first eigenvalue of (1.4) satisfies

$$
\lambda_{1}(h) \geq \mu_{1}(h)
$$

and the equality sign holds if $m=\sqrt{\rho}, \rho$ is given by (5.16) and $\Omega$ is an annulus of radii $R_{1}>0$ and $R_{2}=\frac{1}{\sqrt{\gamma}}$, with Robin boundary conditions for $r=R_{1}$ and Neumann boundary conditions for $r=R_{2}$.

Remark 5.1. The change of variable $\sigma=\sin (\sqrt{K} s)$ transforms (5.26) into

$$
\left\{\begin{array}{l}
X^{\prime \prime}-\sqrt{K} \cdot \tan (\sqrt{K} s) X^{\prime}+\mu X=0 \text { in }\left(0, s_{0}\right) \\
X^{\prime}(0)=0, X^{\prime}\left(s_{0}\right)+\widetilde{h} \cdot X\left(s_{0}\right)=0, s_{0}=\frac{1}{\sqrt{K}} \cos ^{-1}\left(e^{-K p_{m}}\right) .
\end{array}\right.
$$

In this form the limiting case $K \rightarrow 0$ becomes obvious and it is also evident that an upper bound for $p_{m}$ may be used. 
Remark 5.2. Bounds for $p_{m}$ were given by C. Bandle [2]: If $K_{G} \leq K_{m}$ and $K_{m} \cdot A<4 \pi$, then

$$
p_{m} \leq \frac{1}{K_{m}} \log \frac{4 \pi}{4 \pi-K_{m} A} .
$$

The equality sign holds in (5.32) if $\Omega$ is a geodesic circle on a sphere or, equivalently, if $\rho(r)$ is given by (5.16) and $\Omega$ is a disk. Then one finds

$$
p(r)=\frac{1}{4 \gamma} \log \left(\frac{1+\gamma R^{2}}{1+\gamma r^{2}}\right), \quad R=\text { Radius . }
$$

If $K_{G} \geq K>0$ it was shown in [16] that one has

$$
p_{m} \leq \frac{1}{K} \log \frac{1}{\cos (\widehat{d} \sqrt{K})},
$$

where $\widehat{d}$ is the radius of the largest geodesic circle inscribed in $\Omega \subset S$.

If $\Omega$ is an annulus of radii $R, \frac{1}{\gamma R}$ one finds

$$
p_{m}=\frac{1}{K} \log \frac{1}{\cos (\widehat{d} \sqrt{K})}=\frac{1}{4 \gamma} \log \left(\frac{1+\gamma R^{2}}{2 \sqrt{\gamma} R}\right),
$$

so that one has the equality sign in this case.

Using the bound (5.34) one finds that one may take

$$
\sigma_{0}=\sin (\widehat{d} \sqrt{K})
$$

in Theorem 5.1. Note that

$$
\widehat{d} \leq \max _{y \in \partial \Omega}\left(\min _{x \in \Omega} \int_{x}^{y} \sqrt{\rho(s)} d s\right)
$$

and the integral may be taken over a straight line from $x$ to $y$.

\section{Examples}

\subsection{A survey of bounds for the torsion function}

Let $\Omega$ be a plane domain of area $A$ and boundary length $L$. The torsional rigidity $S$ is defined as

$$
S=\int_{\Omega} \psi d x=\int_{\Omega}|\nabla \psi|^{2} d x,
$$

where $\psi$ is the solution of (1.3). 
It was shown by Pólya-Szegö [13] that

$$
\psi_{m} \leq \frac{A}{4 \pi},
$$

with equality for a disk. This inequality was sharpened by Payne [5] who showed that

$$
\psi_{m} \leq \sqrt{\frac{S}{2 \pi}},
$$

which is an improvement of (6.1) since the classical Saint-Venant inequality (proven in [13]) states that

$$
S \leq \frac{A^{2}}{8 \pi}
$$

In (6.2), (6.3) the optimal domain is the circle. A different type of bound was found in [6]: If $\Omega$ is convex then

$$
\psi_{m} \leq \frac{d^{2}}{2}, \quad d=\text { inradius of } \Omega
$$

with equality if $d$ is an infinite strip.

If $\Omega$ is strictly convex with curvature $k \geq k_{0}>0$ then inequality (6.4) can be sharpened. One has (see [16])

$$
\left.\psi_{m} \leq \frac{\tau}{k_{0}} \log \left(\cosh \sqrt{\frac{k_{0}}{\tau}} d\right)\right)
$$

but one needs an upper bound for $\tau=\max _{\partial \Omega}|\nabla \psi|$. Bounds for $\tau$ will be given below.

A number of lower bounds for $\psi_{m}$ are also known. It was shown by Payne [5] that one has

$$
\psi_{m} \geq \frac{1}{4 \pi}\left(A-\sqrt{A^{2}-8 \pi S}\right)
$$

which can be combined with the inequality of Pólya-Szegö [13]

$$
S \geq \frac{A^{2}}{4 B}, B \text { defined in (4.1) }
$$

or the inequality of Payne \& Weinberger [12].

$$
S \geq \frac{A^{2}}{8 \pi}\left[1-\frac{2 \nu}{1-\nu}-\frac{2 \nu^{2}}{(1-\nu)^{2}} \log \nu\right], \nu=1-\frac{4 \pi A}{L^{2}} .
$$

In the last three bounds equality holds again for a disk. A further bound is (see $[2])$

$$
\psi_{m} \geq \frac{\dot{r}^{2}}{4}, \dot{r}=\text { maximal conformal radius of } \Omega \text {. }
$$


Often one can find good bounds for $\psi_{m}$ by exploiting the fact that $\psi_{m}$ depends monotonically on the domain. A bound which becomes sharp in the limit for an infinite strip was also given in [6]: for a convex domain one has

$$
\psi_{m} \geq \frac{A^{2}}{2 L^{2}}
$$

and this can be further improved if $k_{0}>0$. It follows from an inequality of Webb [18] that

$$
\psi_{m} \geq \frac{L-k_{0} A}{2 L-3 k_{0} A} \cdot \frac{A^{2}}{L^{2}}
$$

with equality for a disk or in the limit of an infinite strip.

A bound for $\tau$, the maximum of $|\nabla \psi|$ was first derived by Payne in [6] who showed that for a convex domain

$$
\tau \leq d
$$

with equality for an infinite strip.

If $\Omega$ is strictly convex, i.e. $k \geq k_{0}>0$ then $\mathrm{Fu} \&$ Wheeler [4] proved that

$$
\tau \leq d\left(1-\frac{k_{0} d}{2}\right)
$$

where the equality sign holds for a disk and an infinite strip. The same is true for the bound of Webb [18]

$$
\tau^{2} \leq \psi_{m} \frac{2-3 k_{0} \tau}{1-k_{0} \tau}
$$

\subsection{Some numerical results}

In order to get an idea of how close the bounds for $\lambda_{1}(h)$ are let us first look at a case where most quantities needed are easy to get. This is true e.g. if

\section{A. $\Omega=$ Ellipse of semiaxes $(2,1)$}

The quantities needed are:

$A=2 \pi, L=9.68845, B=\frac{5}{2} \pi, k_{0}=\frac{1}{4}, \psi_{m}=0.4, \tau=0.8$.

For (4.6) the function $\nu(x, y)=1-\frac{x^{2}}{4}-y^{2}$ was taken. Some bounds for $\lambda_{1}(h)$ are displayed in Table 1. 


\begin{tabular}{|c|c|c|c|c|c|c|c|}
\hline \multirow[b]{2}{*}{$h$} & \multicolumn{4}{|c|}{ Lower bounds } & \multicolumn{3}{|c|}{ Upper bounds } \\
\hline & Th.3.1 & Th.3.2 & Th.3.3 & $(3.21)$ & Th.4.1 & $(4.5)$ & $(4.6)$ \\
\hline 0.5 & 0.513 & 0.540 & 0.512 & 0.443 & 0.664 & 0.683 & 0.664 \\
\hline 1 & 0.896 & 0.943 & 0.895 & 0.788 & 1.155 & 1.207 & 1.156 \\
\hline 2 & 1.420 & 1.491 & 1.417 & 1.279 & 1.806 & 1.906 & 1.812 \\
\hline 5 & 2.144 & 2.241 & 2.141 & 1.980 & 2.645 & 2.761 & 2.680 \\
\hline 10 & 2.554 & 2.660 & 2.552 & 2.375 & 3.079 & 3.162 & 3.145 \\
\hline 20 & 2.812 & 2.920 & 2.809 & 2.618 & 3.334 & 3.383 & 3.428 \\
\hline$\infty$ & 3.113 & 3.221 & 3.080 & 2.892 & 3.614 & 3.614 & 3.750 \\
\hline
\end{tabular}

Table 1. Bounds for $\lambda_{1}(h)$ of an ellipse of semiaxes $(2,1)$

\section{Remarks.}

1. The value for $\lambda_{1}(\infty)$ (determined numerically) is $\sim 3.567$. Note how close to this value the entry 3.614 is, which is the bound $j_{0}^{2} \frac{B}{2 A}$ of Pólya-Szegö.

2. The value for $\lambda_{1}(h)$ of the circumscribed rectangle of sides $(4,2)$ is 1.357 and this is larger than the value 1.155 found in Theorem 4.1. This is an example showing that $\lambda_{1}(h)$ does not depend monotonically on the domain.

\section{B. Domain bounded by two arcs}

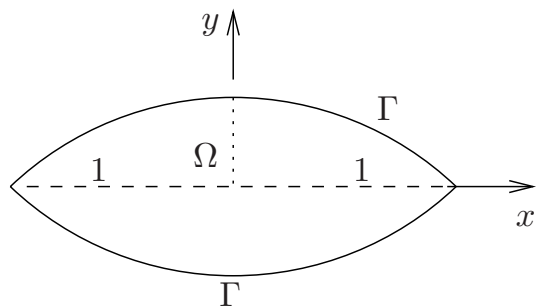

$\Gamma$ : circular arc of radius 2 and length $\frac{2 \pi}{3}$

$$
L=\frac{4 \pi}{3}, A=0.724688, B=4 \pi, k_{0}=\frac{1}{2}
$$

In the following a list of possibilities is given how to get bounds for the required quantities $\psi_{m}$ and $\tau$. Inequalities (6.1) and (6.6) combined with (6.7) give

$$
0.0169 \leq \psi_{m} \leq 0.0577,
$$


whereas (6.11) yields the cruder lower bound $\psi_{m} \geq 0.0157$. Monotonicity with respect to the circumscribed ellipse of semiaxes $a=1, b=2-\sqrt{3}$ gives

$$
\psi_{m} \leq \frac{1}{2\left(\frac{1}{a^{2}}+\frac{1}{b^{2}}\right)}=0.0335,
$$

whereas Payne's bound (6.4) is

$$
\psi_{m} \leq 0.0359,
$$

If we combine the best upper bound for $\psi_{m}$ with Webb's inequality (6.14) we find

$$
\tau \leq 0.2494
$$

whereas Fu \& Wheeler's bound (6.13) yields

$$
\tau \leq 0.25 \text {. }
$$

If the best bound for $\tau$ is used in (6.5) one obtains

$$
\psi_{m} \leq 0.0351 .
$$

Finally the function

$$
v(x, y)=\frac{1}{32}\left(4-x^{2}-(y-\sqrt{3})^{2}\right)\left(4-x^{2}-(y+\sqrt{3})^{2}\right)
$$

satisfies

$$
\Delta v=-1+\frac{1}{2}\left(x^{2}+y^{2}\right) \text { in } \Omega, v=0 \text { on } \partial \Omega,
$$

and hence

$$
\psi_{m}>v_{\max }=\frac{1}{32}=0.03125 .
$$

Also one has

$$
\tau>|\nabla v|_{\max }=0.232 .
$$

If $v(x, y)$ is used in Rayleigh's principle for $\lambda_{1}$ one gets

$$
\lambda_{1} \leq 46.56 \text {. }
$$

The best bounds for $\psi_{m}$ and $\tau$ and $v(x, y)$ in (4.6) were used to get the Table 2 . 


\begin{tabular}{|c|c|c|c|c|c|c|}
\hline \multirow[b]{2}{*}{$h$} & \multicolumn{3}{|c|}{$\lambda_{1}(h) \geq$} & \multicolumn{3}{|c|}{$\lambda_{1}(h) \leq$} \\
\hline & Th.3.1 & Th.3.2 & $(3.21)$ & Th.4.1 & $(4.5)$ & $(4.6)$ \\
\hline 0.5 & 1.91 & 1.85 & 1.96 & 2.75 & 2.82 & 2.77 \\
\hline 1 & 3.67 & 3.55 & 3.70 & 5.33 & 5.51 & 5.30 \\
\hline 2 & 6.76 & 6.56 & 6.63 & 9.84 & 10.44 & 9.74 \\
\hline 5 & 13.59 & 13.24 & 12.34 & 19.75 & 21.78 & 19.29 \\
\hline 10 & 20.23 & 19.81 & 16.93 & 29.17 & 31.91 & 28.01 \\
\hline 20 & 26.45 & 26.04 & 20.43 & 37.53 & 39.11 & 35.49 \\
\hline$\infty$ & 37.02 & 36.83 & 25.1 & 50.14 & 46.56 & 46.56 \\
\hline
\end{tabular}

Table 2. Bounds for $\lambda_{1}(h)$

\section{Oval domain}

A slight deformation of a circle given in polar coordinates as

$$
r=1+0.2 \cdot \cos ^{3} \varphi
$$

is the last example.

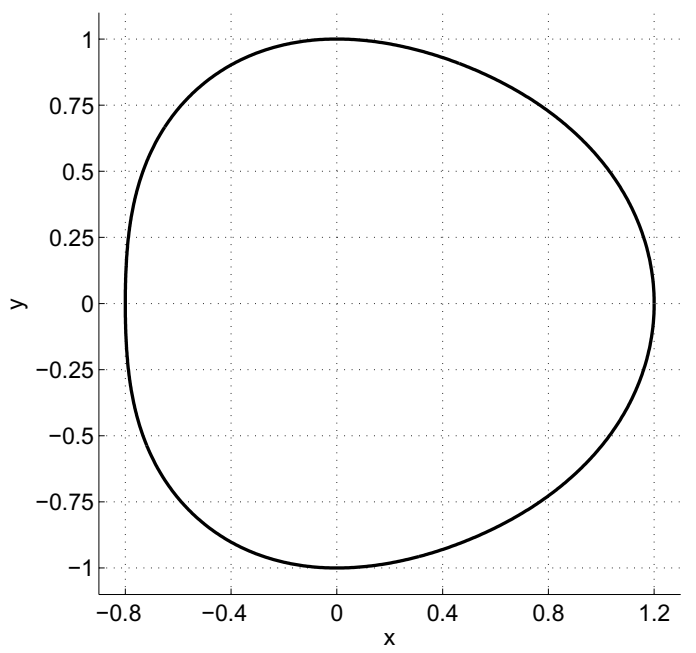

The relevant quantities are

$A=3.18086, L=6.35409, B=6.4303, k_{0}=0.3125, k_{m}=1.25$.

Inequalities (6.1) and (6.6), (6.7) give

$$
0.2148<\psi_{m}<0.2531
$$


and (6.14) then yields

$$
\tau<0.6633
$$

In Table 3 we list the numerical results obtained with these bounds.

\begin{tabular}{c|c|c|c|c|}
\multicolumn{1}{c}{} & \multicolumn{3}{c}{$\lambda_{1}(h) \geq$} & $\lambda_{1}(h) \leq$ \\
$h$ & Th.3.1 & Th.3.2 & $(3.21)$ & Th.4.1 \\
\hline 0.5 & 0.662 & 0.640 & 0.879 & 0.885 \\
1 & 1.186 & 1.149 & 1.565 & 1.579 \\
2 & 1.950 & 1.899 & 2.536 & 2.567 \\
5 & 3.118 & 3.064 & 3.919 & 3.986 \\
10 & 3.847 & 3.804 & 4.697 & 4.790 \\
20 & 4.330 & 4.301 & 5.177 & 5.285 \\
$\infty$ & 4.917 & 4.912 & 5.712 & 5.846
\end{tabular}

\section{References}

[1] M. Abramowitz and I. Stegun, Handbook of mathematical functions. Dover, New York, 1970.

[2] C. Bandle, Isoperimetric inequalities and applications. Pitman, London, 1980.

[3] M. H. Bossel, Longuers extrémales et fonctionelles de Domaine. Complex Variables 6 (1986), 203-234.

[4] S. L. Fu and L. T. Wheeler, Stress bounds for bars in torsion. J. Elasticity 3 (1973), 1-13.

[5] L. E. Payne, Some isoperimetric inequalities in the torsion problem for multiply connected regions. Studies in Math. Anal. and Related Topics: essays in honor of G. Pólya, Stanford Univ. Press, 270-280, 1962.

[6] L. E. Payne, Bounds for the maximum stress in the Saint Venant torsion problem. Ind. J. mech. Math. Special issue (1968), 51-59.

[7] L. E. Payne, Bounds for the solutions of a class of quasilinear elliptic boundary value problems in terms of the torsion function. Proc. Royal Soc. Edinburgh 88A (1981), 251-265.

[8] L. E. Payne and G. A. Philippin, Some remarks on the problems of elastic torsion and of torsional creep. Some Aspects of Mechanics of Continua, Part I. Jadavpur University, 32-40, 1977.

[9] L. E. Payne and G. A. Philippin. Isoperimetric inequalities in the torsion and clamped membrane problem for convex plane domains. SIAM J. Math. Analysis 14 (1983), 11541162.

[10] L. E. Payne and M. E. Rayner, An isoperimetric inequality for the first eigenfunction in the fixed membrane problem Z. angew. Math. Phys. 23 (1972), 13-15.

[11] L. E. Payne and H. F. Weinberger, Lower bounds for vibration frequencies of elastically supported membranes and plates SIAM J. of Appl. Math. 5 (1957), 171-182.

[12] L. E. Payne and H. F. Weinberger, Some isoperimetric inequalities for membrane frequencies and torsional rigidity. J. Math. Anal. Appl. 2 (1961), 210-216.

[13] G. Pólya and G. Szegő, Isoperimetric inequalities in mathematical physics, Princeton University Press, 1952. 
[14] P. W. Schaefer and R. Sperb, Maximum principles for some functionals associated with the solution of elliptic boundary value problems. Arch. Rat. Mech. Anal. 61 (1976), 65-76.

[15] R. Sperb, Obere und untere Schranken für den tiefsten Eigenwert des elastisch gestützten Membran. Z. angew. Math. Phys. 23 (1972), 231-244.

[16] R. Sperb, Maximum principles and their applications. Academic Press, New York, 1981.

[17] R. Sperb, Optimal bounds in semilinear elliptic problems with nonlinear boundary conditions. Z. angew. Math. Phys. 44 (1993), 639-653.

[18] J. R. L. Webb, Maximum principles for functionals associated with the solution of semilinear elliptic boundary value problems. Z. angew. Math. Phys. 40 (1989), 330-338.

R. Sperb

Seminar für Angewandte Mathematik

ETH-Zentrum

CH-8092 Zürich

e-mail: sperb@sam.math.ethz.ch

(Received: June 16, 2003) 